\title{
Comparación de la ganancia y otros parámetros de operación de antenas Yagi alimentadas con un dipolo recto o con un dipolo doblado
}

\author{
R. Neri-Vela, L.A. Valiente-Montaño y V. Hernández-Solís \\ Departamento de Telecomunicaciones, Facultad de Ingeniería, UNAM \\ E-mail: aldeca@marconi.fi-b.unam.mx
}

(recibido: abril de 2005; aceptado: agosto de 2005)

\begin{abstract}
Resumen
La ganancia y otras características de operación de las antenas Yagi están estrechamente relacionadas con la posición y orientación de su elemento alimentado, y no solamente con el número de elementos radiantes o el espaciamiento entre éstos. En este artículo se presenta una comparación entre Yagis alimentadas con un dipolo recto sencillo y con un dipolo doblado. En este último caso, se analizan configuraciones coplanares con alimentación en el brazo izquierdo o en el brazo derecho, y también se estudia qué sucede cuando el dipolo doblado es transversal al plano que contiene a todos los demás elementos del arreglo. Los resultados mostrados son de utilidad práctica y académica, ya que la mayoría de estos detalles técnicos de operación no se encuentran en la literatura científica, a pesar de que estas antenas aún son utilizadas en una gran variedad de aplicaciones comerciales.
\end{abstract}

Descriptores: Antena Yagi, dipolo doblado, método de momentos.

\begin{abstract}
Thegain and other op er a tional prop er ties of a Yagi an tenna areclosely related to the po si tion and orien ta tion of its fed el ement, and not just to thenum ber of its ra di at ing el ements or thespacingbetween them. A com par i son between sin gle dipole fed and folded-dipole fed an ten nas is presented in this paper, con sid er ing coplanar right-arm-fed, coplanar left-arm-fed and tran sversal con fig u ra tions for the folded di pole. The re sults shown are use ful in the de sign and un der stand ing of this type of antenna when fed with a folded di pole, sinceal most no per for manceval ues arefound in thetech ni cal liter ature. Thepro cedure fol lowed to ob tain them should beuseful for stu dents and teach ers on thesubject of lin ear an ten nas, which arestill widely used in a va ri ety of com mer cial ap pli ca tions.
\end{abstract}

Keywords: Yagi an tenna, folded di pole, mo ment method.

\section{Introducción}

El arreglo Yagi-Uda clásico, inventado de forma empírica hace aproximadamente ocho décadas y más comúnmente conocido como antena Yagi, es utilizado actualmente en una gran variedad de aplicaciones en ingeniería; entre éstas se encuentran la recepción de señales de TV abierta en VHF, así como la recepción de TV de alta definición
(HDTV) en UHF (Neri y Valiente, 2003), transmisión de datos punto a punto, estaciones de radioaficionados en todo el mundo (Neri et al., 2005), comunicaciones rurales y telefonía celular, telemetría y comunicaciones espaciales (Landeros et al., 2005), por mencionar sólo algunas. Esta antena es un radiador práctico empleado en las bandas de $\mathrm{HF}$ (3-30 MHz), VHF (30-300 MHz) y UHF (300-3000 $\mathrm{MHz}$ ), y usualmente consiste de un reflector 
Comparación de la ganancia y otros parámetros de operación de antenas Yagi ...

parásito, un elemento alimentado y varios directores parásitos (Balanis, 1997). El estudio de este tipo de antenas es obligado en cualquier curso de ingeniería de telecomunicaciones a nivel licenciatura y, a pesar de que una gran cantidad de autores ha realizado exhaustivas investigaciones analíticas y experimentales publicadas en libros de texto reconocidos mundialmente (Balanis, 1997; Kraus, 1988; Combes, 1997; Stutzman et al., 1998 y Griffiths, 1987) así como en prestigiadas revistas técnicas (Ehrenspeck et al., 1959; Mailloux, 1966; Thiele, 1969; Cheng et al., 1973; Chen et al., 1975 y King, 1989), aún existen detalles importantes por de terminar. En partic u lar, aun cuando el estudiante puede saber que la impedancia de entrada de la antena puede ser modificada utilizando un dipolo doblado en lugar de un dipolo recto sencillo como elemento alimentado, encontrará que algunos muestran el dipolo doblado transversal al plano del arreglo (Kraus, 1988 y Stutzman et al., 1998), mientras que otros lo presentan coplanar a éste (Combes, 1997 y Griffiths, 1987), sin aclarar en este último caso, cuál lado del dipolo doblado (izquierdo o derecho) es mejor para conectarlo con la línea de transmisión, o si el resultado es el mismo en ambas situaciones (Figuras 1 y 2). A conocimiento de los autores de este artículo, ninguna fuente comenta sobre si la orientación del dipolo doblado tiene relevancia o no, aunque el dipolo doblado es por sí mismo una antena directiva con ganancia máxima cercana a los $2.7 \mathrm{dBi}$, dependiendo del espaciamiento entre sus brazos (Hernández et al, 2001), y su patrón de radiación, en el caso de dipolos doblados con espaciamientos prácticos, no es "exactamente igual" al de un dipolo recto sencillo, tal y como lo han expresado muy someramente algunos autores (Gibilisco, 1999). La figura 1 muestra una comparación entre los patrones de radiación en el plano $E$ de un dipolo recto de longitud $\lambda / 2$ alimentado en su centro y un dipolo doblado alimentado en su brazo izquierdo (Hernández et al., 2001); dado que estos patrones no son exactamente iguales, es de esperarse, que al utilizar uno $u$ otro tipo de dipolo como alimentador en un arreglo Yagi, contribuya de manera distinta al acoplamiento de impedancias mutuas con los elementos parásitos y, por tanto, al patrón de radiación total y demás características de operación.

Además de los autores mencionados anteriormente, otros más han ilustrado al dipolo doblado con ambas orientaciones, coplanar y transversal (Jasik, 1961), pero la mayoría restante no presenta un dibujo del arreglo, a pesar de que el dipolo doblado también ayuda a mejorar el ancho de banda (Cardama et al., 2004), por lo que el estudiante se ve forzado a asumir una orientación arbitraria. Adicionalmente, aun después de aseverar que "el elemento alimentador más común para una antena Yagi-Uda es un dipolo doblado" (Balanis, 1997), un buen número de los libros de texto se concentra en proporcionar una gran cantidad de datos sobre arreglos Yagi alimentados con un dipolo recto sencillo, incluyendo ganancia y patrones de radiación, pero nunca comentan sobre estos parámetros para arreglos alimentados con un dipolo doblado. En rarísimas ocasiones, simplemente se le asegura al lector que el uso del dipolo doblado en lugar de un dipolo recto sencillo, no afecta al resto de los parámetros del arreglo
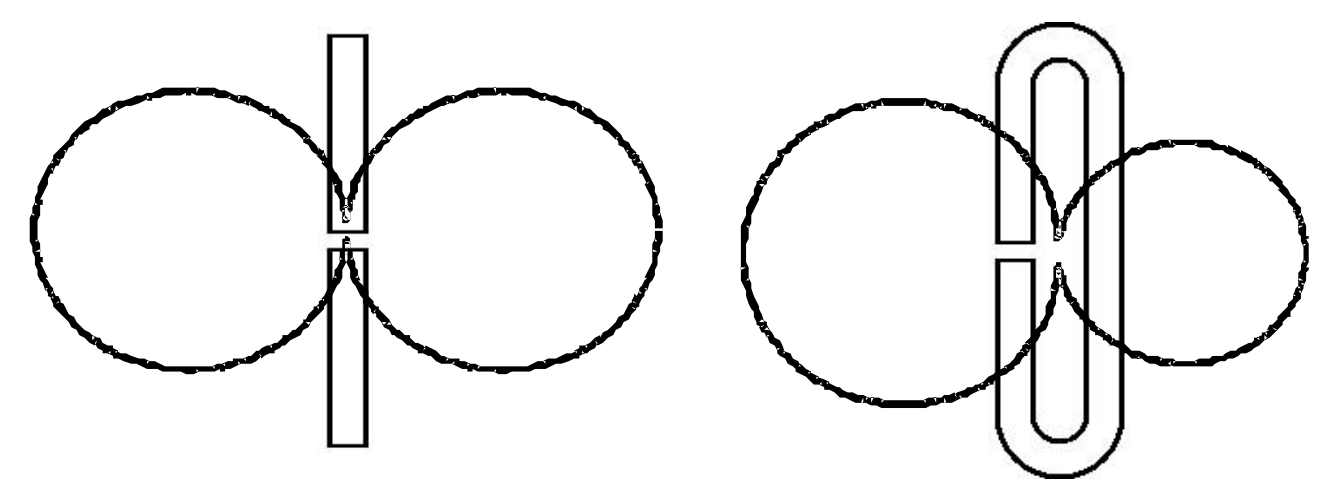

Figura 1. Patrones de radiación en el plano E para un dipolo sencillo y un dipolo doblado ( $H$ ernández et al., 2001). El pequeño hueco en el conductor indica el punto de alimentación. A mbos dipolos tien en un grosor de $0.0166 \lambda$ y una

longitud 0 altura de $\lambda / 2$. La separación entre los dos brazos del dipolo doblado es de $0.15 \lambda$ 
R. Neri-Vela, L.A. Valiente-Montaño y V. Hernández-Solís

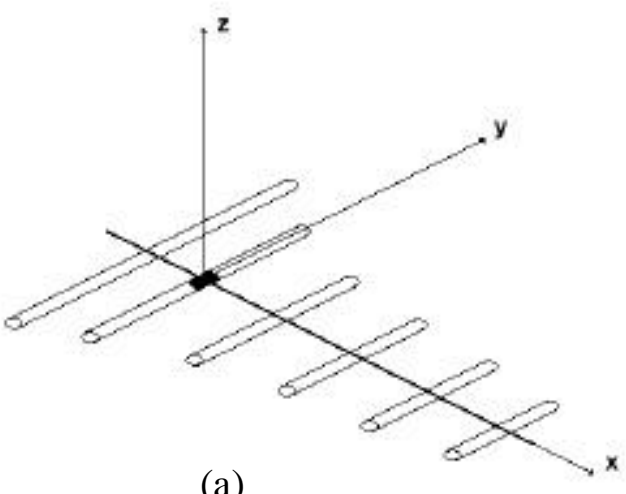

(a)

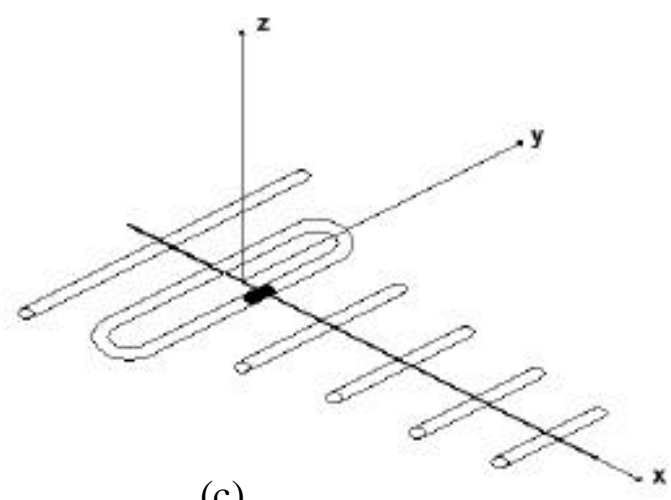

(c)

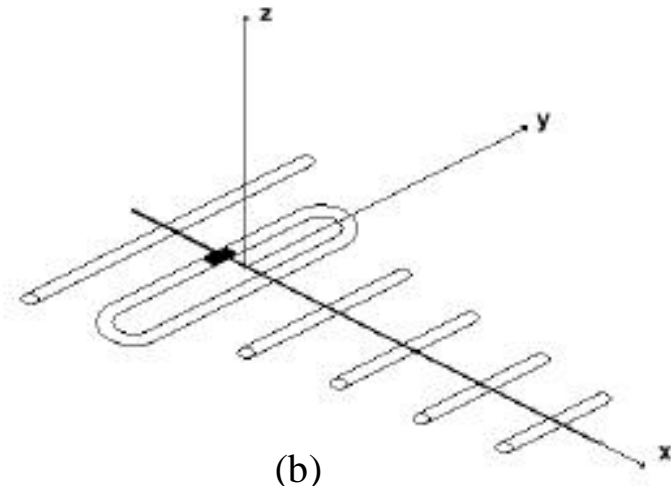

(b)

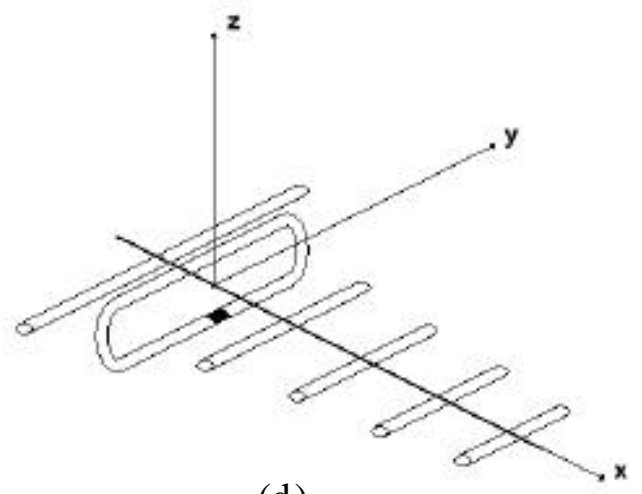

(d)

Figura 2. Configuraciones de arreglos Yagi consideradas en este estudio, mostradas para el caso de 6 elementos: a) Alimentación con dipolo recto sencillo, b) Alimentación con dipolo doblado coplanar y con la línea de transmisión conectada del lado izquierdo, c) Alimentación con dipolo doblado coplanar y con la línea de transmisión conectada del lado derecho; y d) Alimentación con dipolo doblado transversal y centrado en el origen. Los ejes de todos los reflectores, los directores y los dipolos doblados coplanares están en el plano x-y. El segmento usado como punto de alimentación se muestra oscurecido en cada uno de los arreglos

(Balanis, 1997), pero, nuevamente, esto no indica hasta qué grado, ya que no se demuestra teóricamente, y tampoco se presentan datos experimentales o numéricos al respecto.

Este artículo tiene la intención de servir como una herramienta práctica y didáctica que ayude a aclarar la situación antes mencionada, así como proporcionar información sobre el efecto del tipo de alimentador en la operación del arreglo. Se presentan resultados para arreglos Yagi, cuyo elemento alimentado es:

a) Un dipolo recto sencillo;

b) Un dipolo doblado coplanar, alimentado en el lado más cerca del parásito reflector; c) Un dipolo doblado coplanar, alimentado en el lado más cerca de los parásitos directores; $y$

d) Un dipolo doblado transversal al plano que contiene todos los elementos parásitos.

Estas cuatro configuraciones se muestran en la figura 2.

\section{Modelado y simulación numérica}

Los cuatro casos fueron modelados para ser analizados mediante el conocido método de momentos (Har ring ton, 1968; Tsaiet al., 1978; Neri, 1980 y Wang, 1990). Las variables de longitud, diámetro y separación ilustradas en la figura 3 se 
Comparación de la ganancia y otros parámetros de operación de antenas Yagi ...

muestran en la tabla 1, así como la segmentación de todos los elementos; $\Delta \mathrm{l}$, la longitud de cada segmento, mide aproximadamente $\lambda 10$, y su valor exacto depende del elemento al que pertenece; todos los segmentos rectos tienen la misma longitud en cada elemento in $\mathrm{d} i$ vidual. La curvatura en ambos extremos del dipolo doblado fue modelada exactamente de la misma manera como se hizo en (Hernández et al., 2001). El re flec tor y to dos los directores tienen en los extremos semisegmentos de longitud $\Delta / / 2$ y corriente nula, de acuerdo con los requerimientos del método de momentos; para familiarizarse con el procedimiento para calcular la matriz de impedancias a invertir, se invita al lector a consultar la referencia (Neri, 1980).

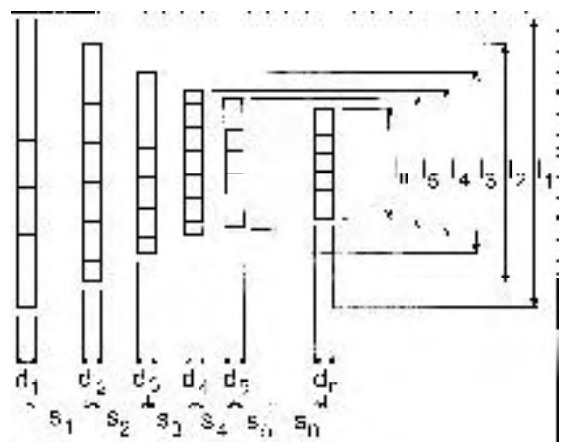

(a)
Las ecuaciones no se incluyen en este trabajo, debido a limitaciones de espacio de impresión. Los autores consideran de mayor importancia utilizar el espacio disponible para mostrar y comentar los resultados obtenidos. Sin embargo, el lector interesado en conocer el procedimiento matemático puede consultar el sitio [http://telecom.fi-b.unam. $\mathrm{mx} /$ web link/The Method of Mo ments.htm], diseñado por los mismos autores. Este sitio también contiene un programa de computadora para el análisis de estas antenas.

En total, se analizaron 60 arreglos Yagi (una muestra suficientemente grande) con los parámetros mostrados en la tabla 1; la longitud de todos los elementos directores es de aproximadamente $0.4 \lambda$, de acuerdo con resultados óptimos

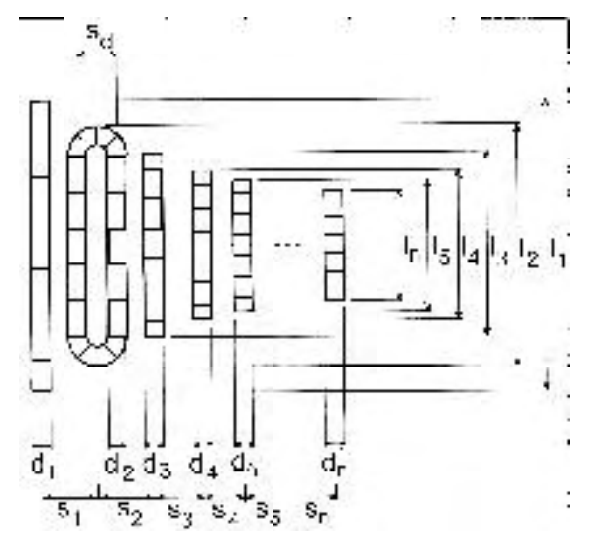

(b)

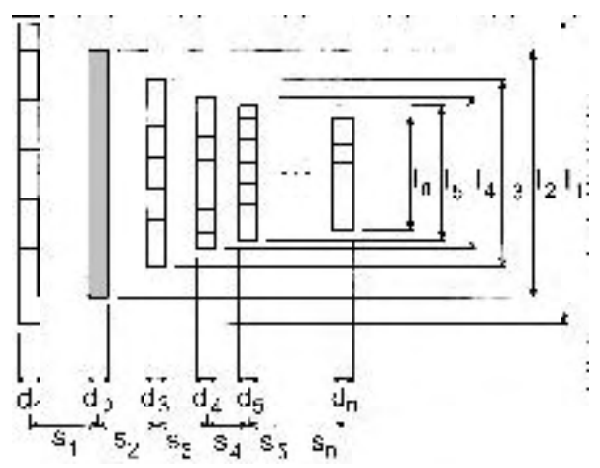

(c)

Figura 3. Vari ables de longitud, diámetro y separación utilizadas en el análisis por el método de momentos. a) Alimentación con dipolo recto sencillo ( $2^{\circ}$ elemento), b) Alimentación con dipolo doblado coplana r; y c) Alimentación con dipolo doblado transversal. Todos los el ementos se muestran segmentados de acuerdo con los requerimientos del método de momentos, la segmentación utlizada para el dipolo doblado coplanar es la misma que en el dipolo transversal, solo que

éste último se muestra en gris, debido a la dificultad de representarlo en forma segmentada (Ver Figura 2d) 
reportados con anterioridad utilizando un dipolo recto sencillo (Chen et al., 1975) o un dipolo doblado transversal (Johnson, 1993), pero el espaciamiento entre ellos, también de aproximadamente $0.4 \lambda$, es ligeramente mayor al sugerido en estas mismas referencias; estos parámetros fueron obtenidos mediante un proceso iterativo de optimización para obtener una ganancia máxima. De acuerdo con (Balanis, 1997), la ganancia de la antena Yagi varía siguiendo un patrón oscilatorio con pequeñas variaciones entre mínimos y máximos al aumentar la separación entre los directores, por lo que existen diversos valores de separación s para valores si- milares de ganancia. Por tanto, es válido utilizar los espaciamientos considerados en este estudio.

Para cada uno de los casos, una vez obtenida la separación ideal spara obtener una ganancia máxima, se indujo la resonancia a $500 \mathrm{MHz}$ ajustando el espaciamiento $s_{1}$ entre el reflector y el elemento alimentado, y variando la longitud del elemento alimentado, con el fin de reducir o incrementar su reactancia de entrada hasta obtener un valor cercano a cero $(\leq 1 \Omega)$. Con esto se garantiza la posibilidad de un acoplamiento óptimo con la línea y se pueden comparar correctamente los resultados de ganancia.

Tabla 1. A rreglos Yagi-U da con un dipolo resonante como elemento alimentado. Todos los elementos, incluyendo al dipolo doblado, tienen el mismo diámetro $(0.0166 \lambda)$. La separación $s_{d}$ entre los ejes de ambos brazos del dipolo doblado es de $0.0833 \lambda$ para todos los casos, y en cada arreglo todos los directores tienen la misma longitud eléctrica $\left(\right.$ ó $I_{3}$ hasta $\left.I_{n}\right)$. Las separaciones $s_{2}$ a $s_{n}$ de la figura 3 son iguales a $s$

Yagi con dipolo sencillo

Yagi con dipolo doblado transversal

\begin{tabular}{|c|c|c|c|c|c|c|c|c|c|c|c|c|c|c|c|c|}
\hline $\begin{array}{l}\text { Número de } \\
\text { elementos }\end{array}$ & $\left.I_{1} \lambda\right]$ & $b[\lambda]$ & $\mid[\lambda]$ & $\left.s_{1} \lambda\right]$ & $\$ \lambda]$ & $\mathrm{G}[\mathrm{dBi}]$ & $z[\Omega]$ & $\mathrm{FBR}[\mathrm{dB}]$ & $\mathrm{I}_{1}[\lambda]$ & $\left.\right|_{2}[\lambda]$ & $\mid Q]$ & $S[]$ & $S_{n}[\lambda]$ & $\mathrm{G}[\mathrm{dBi}]$ & $\mathrm{Z}_{\mathrm{i}}[\Omega]$ & FBR [dB] \\
\hline 5 & 0.47 & 0.431 & 0.4 & 0.2 & 0.4 & 10.7 & 21.9 & 7.3 & 0.49 & 0.4 & 0.4 & 0.19 & 0.4 & 11.1 & 86.8 & 8.4 \\
\hline 6 & 0.47 & 0.434 & 0.4 & 0.2 & 0.4 & 11.5 & 21.4 & 11.5 & 0.49 & 0.405 & 0.4 & 0.19 & 0.4 & 12.1 & 86.2 & 12.2 \\
\hline 7 & 0.47 & 0.438 & 0.4 & 0.2 & 0.4 & 12.1 & 24.1 & 20.5 & 0.49 & 0.411 & 0.4 & 0.19 & 0.4 & 12.7 & 102.8 & 23.7 \\
\hline 8 & 0.47 & 0.437 & 0.41 & 0.22 & 0.39 & 12.5 & 25.9 & 23.6 & 0.47 & 0.425 & 0.41 & 0.17 & 0.39 & 13.6 & 77.1 & 14.4 \\
\hline 9 & 0.47 & 0.437 & 0.41 & 0.22 & 0.39 & 12.5 & 33.3 & 11.4 & 0.47 & 0.418 & 0.41 & 0.17 & 0.39 & 13.6 & 101.1 & 8.3 \\
\hline 10 & 0.47 & 0.431 & 0.41 & 0.22 & 0.39 & 12.4 & 35.7 & 7.6 & 0.47 & 0.407 & 0.41 & 0.17 & 0.39 & 13.7 & 81.7 & 6.3 \\
\hline 11 & 0.47 & 0.436 & 0.4 & 0.19 & 0.39 & 13.6 & 21.0 & 14.0 & 0.47 & 0.411 & 0.4 & 0.19 & 0.39 & 14.5 & 73.2 & 15.1 \\
\hline 12 & 0.47 & 0.440 & 0.4 & 0.19 & 0.39 & 13.8 & 22.7 & 24.7 & 0.47 & 0.417 & 0.4 & 0.19 & 0.39 & 14.6 & 87.5 & 27.4 \\
\hline 13 & 0.47 & 0.440 & 0.4 & 0.19 & 0.39 & 13.9 & 26.7 & 14.0 & 0.47 & 0.417 & 0.4 & 0.19 & 0.39 & 14.6 & 112.6 & 12.4 \\
\hline 14 & 0.47 & 0.437 & 0.4 & 0.18 & 0.38 & 14.1 & 21.1 & 12.0 & 0.48 & 0.401 & 0.4 & 0.2 & 0.38 & 14.8 & 104.2 & 10.5 \\
\hline 15 & 0.47 & 0.440 & 0.4 & 0.18 & 0.38 & 14.3 & 19.6 & 19.8 & 0.48 & 0.405 & 0.4 & 0.2 & 0.38 & 15.3 & 88.1 & 14.4 \\
\hline
\end{tabular}

\begin{tabular}{|c|c|c|c|c|c|c|c|c|c|c|c|c|c|c|c|c|}
\hline \multirow[b]{2}{*}{$\begin{array}{l}\text { Número de } \\
\text { elementos }\end{array}$} & \multicolumn{8}{|c|}{ Yagi con dipolo doblado coplanar (alimentación izquierda) } & \multicolumn{8}{|c|}{ Yagi con dipolo doblado coplanar (alimentación derecha) } \\
\hline & $\left.I_{1} \lambda\right]$ & $b[\lambda]$ & $\mid[\lambda]$ & $\left.s_{1} \lambda\right]$ & $\$ \lambda]$ & $\mathrm{G}[\mathrm{dBi}]$ & $z[\Omega]$ & $\mathrm{FBR}[\mathrm{dB}]$ & $\mathrm{I}_{1}[\lambda]$ & $\left.\right|_{2}[\lambda]$ & $\mid \mathrm{l}]$ & $\left.s_{1} \lambda\right]$ & $S[\lambda]$ & $\mathrm{G}[\mathrm{dBi}]$ & $\mathrm{Z}_{\mathrm{i}}[\Omega]$ & FBR [dB] \\
\hline 5 & 0.49 & 0.400 & 0.4 & 0.19 & 0.4 & 11.0 & 73.0 & 8.9 & 0.49 & 0.404 & 0.4 & 0.19 & 0.4 & 11.2 & 90.4 & 8.3 \\
\hline 6 & 0.49 & 0.405 & 0.4 & 0.19 & 0.4 & 12.0 & 74.6 & 13.5 & 0.49 & 0.41 & 0.4 & 0.19 & 0.4 & 12.2 & 89.6 & 12.2 \\
\hline 7 & 0.49 & 0.410 & 0.4 & 0.19 & 0.4 & 12.5 & 90.4 & 28.8 & 0.49 & 0.417 & 0.4 & 0.19 & 0.4 & 12.7 & 107.6 & 23.2 \\
\hline 8 & 0.47 & 0.426 & 0.41 & 0.17 & 0.39 & 13.3 & 62.4 & 12.2 & 0.47 & 0.431 & 0.41 & 0.17 & 0.39 & 13.5 & 85.4 & 14.6 \\
\hline 9 & 0.47 & 0.417 & 0.41 & 0.17 & 0.39 & 13.4 & 75.6 & 7.4 & 0.47 & 0.426 & 0.41 & 0.17 & 0.39 & 13.6 & 114.5 & 8.3 \\
\hline 10 & 0.47 & 0.410 & 0.41 & 0.17 & 0.39 & 13.5 & 58.4 & 6.0 & 0.47 & 0.415 & 0.41 & 0.17 & 0.39 & 13.8 & 94.3 & 6.0 \\
\hline 11 & 0.47 & 0.413 & 0.4 & 0.19 & 0.39 & 14.3 & 60.9 & 16.4 & 0.47 & 0.417 & 0.4 & 0.19 & 0.39 & 14.5 & 80.3 & 14.8 \\
\hline 12 & 0.47 & 0.417 & 0.4 & 0.19 & 0.39 & 14.5 & 73.8 & 21.8 & 0.47 & 0.423 & 0.4 & 0.19 & 0.39 & 14.6 & 95.2 & 28.7 \\
\hline 13 & 0.47 & 0.415 & 0.4 & 0.19 & 0.39 & 14.4 & 92.5 & 11.2 & 0.47 & 0.424 & 0.4 & 0.19 & 0.39 & 14.6 & 123.0 & 12.7 \\
\hline 14 & 0.48 & 0.400 & 0.4 & 0.2 & 0.38 & 14.7 & 87.2 & 10.8 & 0.48 & 0.406 & 0.4 & 0.2 & 0.38 & 14.9 & 109.4 & 10.4 \\
\hline 15 & 0.48 & 0.406 & 0.4 & 0.2 & 0.38 & 15.2 & 76.9 & 15.7 & 0.48 & 0.411 & 0.4 & 0.2 & 0.38 & 15.3 & 92.9 & 14.3 \\
\hline
\end{tabular}


Comparación de la ganancia y otros parámetros de operación de antenas Yagi ...

\section{Resultados}

\section{a) Distribuciones de corriente}

Los arreglos Yagi alimentados con un dipolo recto sencillo tienen un máximo de magnitud de corriente precisamente en el centro de dicho elemento (Balanis, 1997 y Thiele, 1969). Los valores máximos de corriente en los directores son menores y no son iguales entre sí, ni disminuyen gradualmente en la dirección de radiación; sus valores máximos y el margen de oscilación dependen de la combinación de longitudes y espaciamientos, así como del número total de directores; no obstante, se presentan los resultados para arreglos de 15 y 27 elementos, como ejemplo. Para el arreglo de 15 elementos (Balanis, 1997), el valor máximo promedio de cualquiera de las 13 corrientes en los directores es aproximadamente del $35 \%$ de la corriente máxima en el punto de alimentación, mientras que el re flector tiene una corriente máxima de sólo el $50 \%$, ya que al aumentar la longitud del arreglo, las corrientes inducidas en los directores disminuyen. Para el arreglo de 27 elementos (Thiele, 1969) el valor máximo promedio para las corrientes en los directores cae a un $20 \%$ en comparación con la corriente máxima en el dipolo alimentado, mientras que la corriente en el reflector tiene un valor máximo mucho mayor que el de los directores en ambos arreglos, equivalente al $50 \%$ y $70 \%$ respectivamente, para los arreglos de 15 y 27 elementos. No se hace aquí ninguna comparación para arreglos con menos de 15 elementos, ya que los autores desconocen si existen datos publicados para estos casos en otras fuentes.

La situación de los valores promedio máximos para las am pli tudes de corriente en los centros de cada con duc tor del arreglo, es distinta cuando se utiliza un dipolo doblado como elemento excitado, lo cual no se ha discutido previamente en la literatura. Las figuras 4 y 5 muestran las am pli tudes máximas relativas de corriente para las cuatro configuraciones consideradas en este artículo en arreglos resonantes de 15 elementos. Es interesante resaltar que, cuando se utiliza un dipolo doblado, ya sea coplanar o transversal al plano del arreglo, el valor máximo de corriente ya no se encuentra en el elemento alimentado sino en el reflector; sin embargo, cada brazo del dipolo doblado tiene una magnitud significativa de corriente, y si se sumaran entre sí, el valor resultante sería nuevamente el máximo, de forma similar al caso discutido al inicio de esta sección. Independientemente de este fenómeno, cuando se utiliza un dipolo doblado coplanar, su brazo izquierdo presenta siempre una distribución de corriente con un valor máximo ligeramente mayor al encontrado en el brazo derecho, sin importar en cual de estos brazos se alimenta al dipolo, como se muestra en la figura 5. Si el dipolo doblado es transversal, los picos de corriente en ambos brazos son más cercanos entre sí, casi iguales, sin llegar a serlo por la pequeña asimetría geométrica del arreglo (Figura 2d); esta misma asimetría, producida por la ubicación del punto de alimentación, es causante de una leve desviación del haz prin ci pal de radiación, que será explicada a detalle más adelante. Este mismo comportamiento relativo de las corrientes, fue observado en arreglos de 5 hasta 14 elementos en las investigaciones realizadas por los autores.

\section{b) Impedancia de entrada}

Como se mencionó anteriormente, todos los arreglos fueron diseñados para resonar a una frecuencia de $500 \mathrm{MHz}$. La resistencia de entrada correspondiente a esta frecuencia se muestra en la tabla 1. Como era de esperarse, el uso de un dipolo doblado en lugar de uno recto, incrementa la impedancia de entrada de cada uno de los arreglos, pero este incremento no es siempre con un fac tor de proporcionalidad cercano a cuatro, de acuerdo con la predicción teórica para dipolos doblados cuyos brazos se encuentran extremadamente cercanos entre sí (Stutzman et al., 1998) y que además son imprácticos (Hernández et al., 2001). Los resultados presentados en la tabla 1 muestran que, en resonancia, la impedancia de entrada de estos arreglos varía entre 75 y $100 \mathrm{O}$, con unas cuantas excepciones para valores ligeramente mayores. Si se considera como alimentación una línea de transmisión con una impedancia característica de $75 \mathrm{O}$, y se mantienen fijos los parámetros físicos del arreglo con los valores del estado de resonancia, las curvas de VSWR resultantes en función de la frecuencia son las mostradas en las figuras 6 y 7 para arreglos de 5 y 15 elementos. Las bandas de frecuencia de 
R. Neri-Vela, L.A. Valiente-Montaño y V. Hernández-Solís

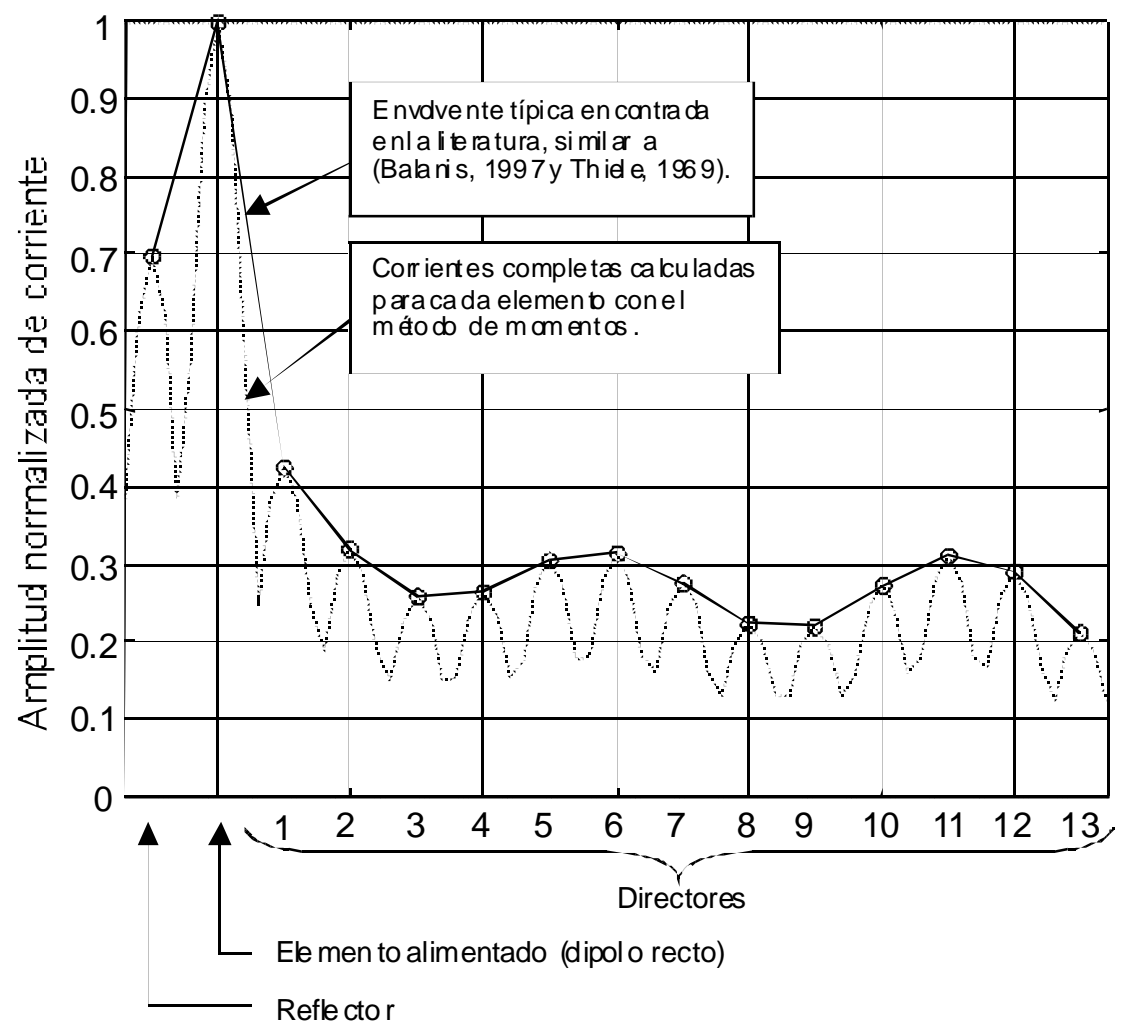

Figura 4. A mplitud normalizada de corriente para una antena Yagi de 15 el ementos, alimentada con un dipolo recto

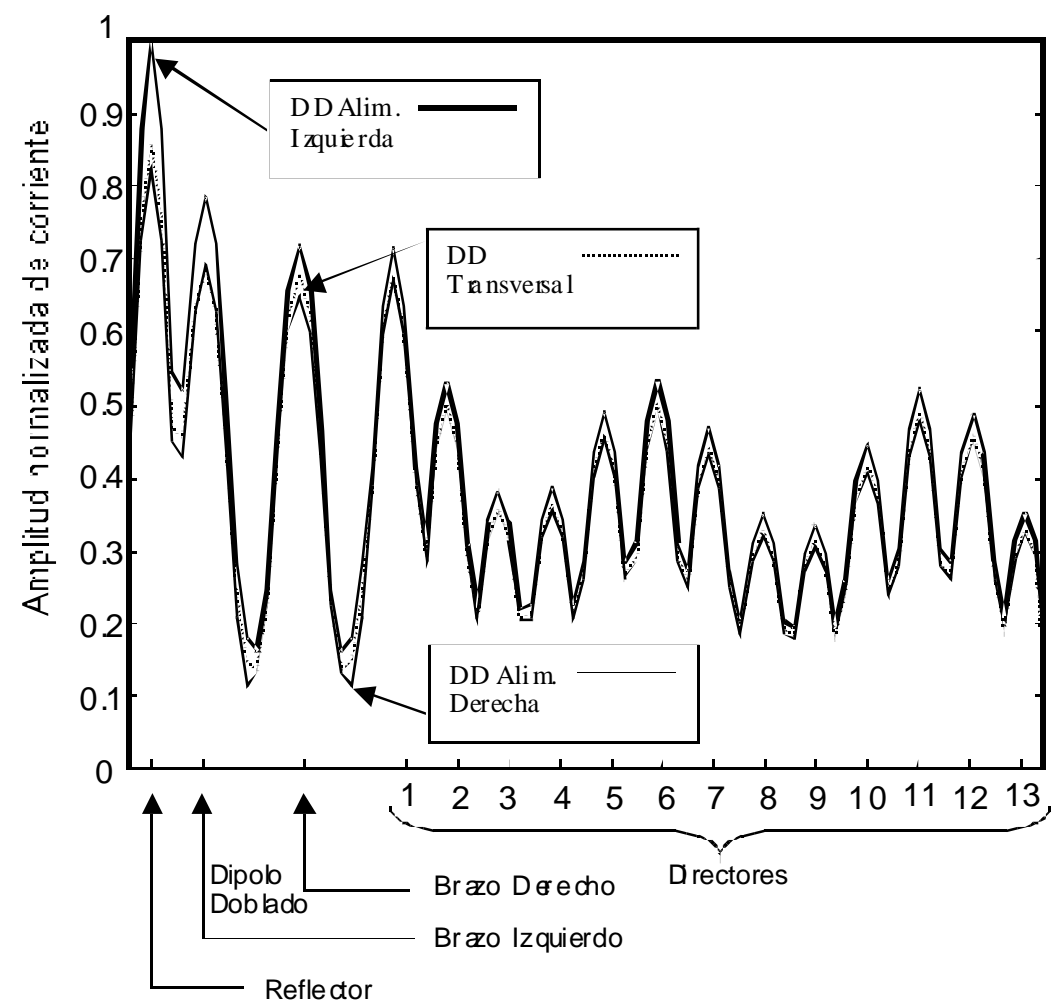

Figura 5. A mplitud normalizada de corriente para antenas Yagi de 15 elementos, alimentadas con dipolos doblados, y calculada con el método de momentos 
Comparación de la ganancia y otros parámetros de operación de antenas Yagi ...

transmisión de TV en UHF para los ca na les 16 al 21 en EUA, se muestran también como referencia.

Evidentemente, estos arreglos son de banda ancha, en el sentido de que varios canales de 6 $\mathrm{MHz}$ pueden ser recibidos con un nivel aceptable de relación de onda estacionaria (VSWR por sus siglas en inglés) menor o igual a $2(0.5 \mathrm{~dB}$ de pérdidas por retorno). La ganancia correspondiente para estos mismos arreglos se discute más adelante en otra sección. Aunque la curva de VSWR es casi la misma para los tres tipos de alimentación con dipolo doblado, es interesante notar que el uso de un dipolo doblado alimentado en su brazo izquierdo tiene resultados ligeramente mejores, mientras que el "peor" de los tres arreglos se obtiene alimentando el dipolo doblado en su brazo derecho, como se observa en las curvas de las figuras 6 y 7 . Asimismo, el efecto de mejorar el ancho de banda es evidente cuando se usa un dipolo doblado en lugar de uno recto (Cardama et al., 2004). Para antenas con un número total de 6 a 14 elementos, se obtiene un comportamiento similar, pero la pendiente de las curvas y el ancho de banda total dependen del número de elementos. En cuanto a las antenas alimentadas con un dipolo recto, se confirma en las figuras que trabajan de manera muy deficiente, en comparación con los arreglos alimentados con un dipolo doblado; esto demuestra que las antenas Yagi no operan de "igual" manera sin importar cómo son alimentadas, tal como se indica en varios textos y cómo se comentó en la introducción de este artículo. El VSWR de las antenas alimentadas con un dipolo recto es muy deficiente, debido al hecho de que su resistencia de entrada es muy baja en comparación con los 750 de la línea de transmisión, aun cuando sean resonantes a $500 \mathrm{MHz}$.

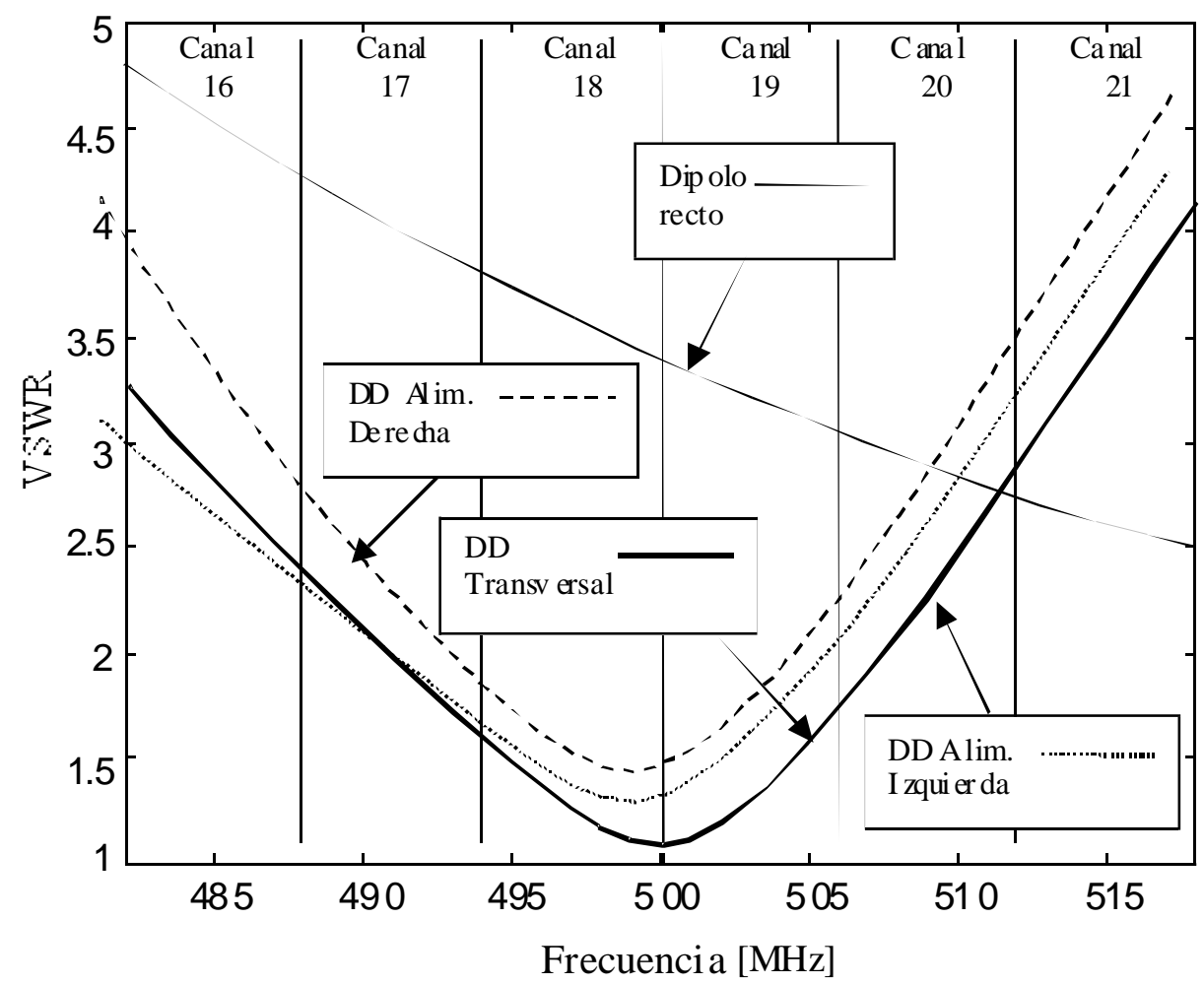

Figura 6. VSWR de arreglos Yagi de 5 elementos, considerando una alimentación con una línea de transmisión de $75 \Omega$. Véase la resistencia de entrada en estado de resonancia a $500 \mathrm{M} \mathrm{Hz}$ en la tabla 1 
R. Neri-Vela, L.A. Valiente-Montaño y V. Hernández-Solís

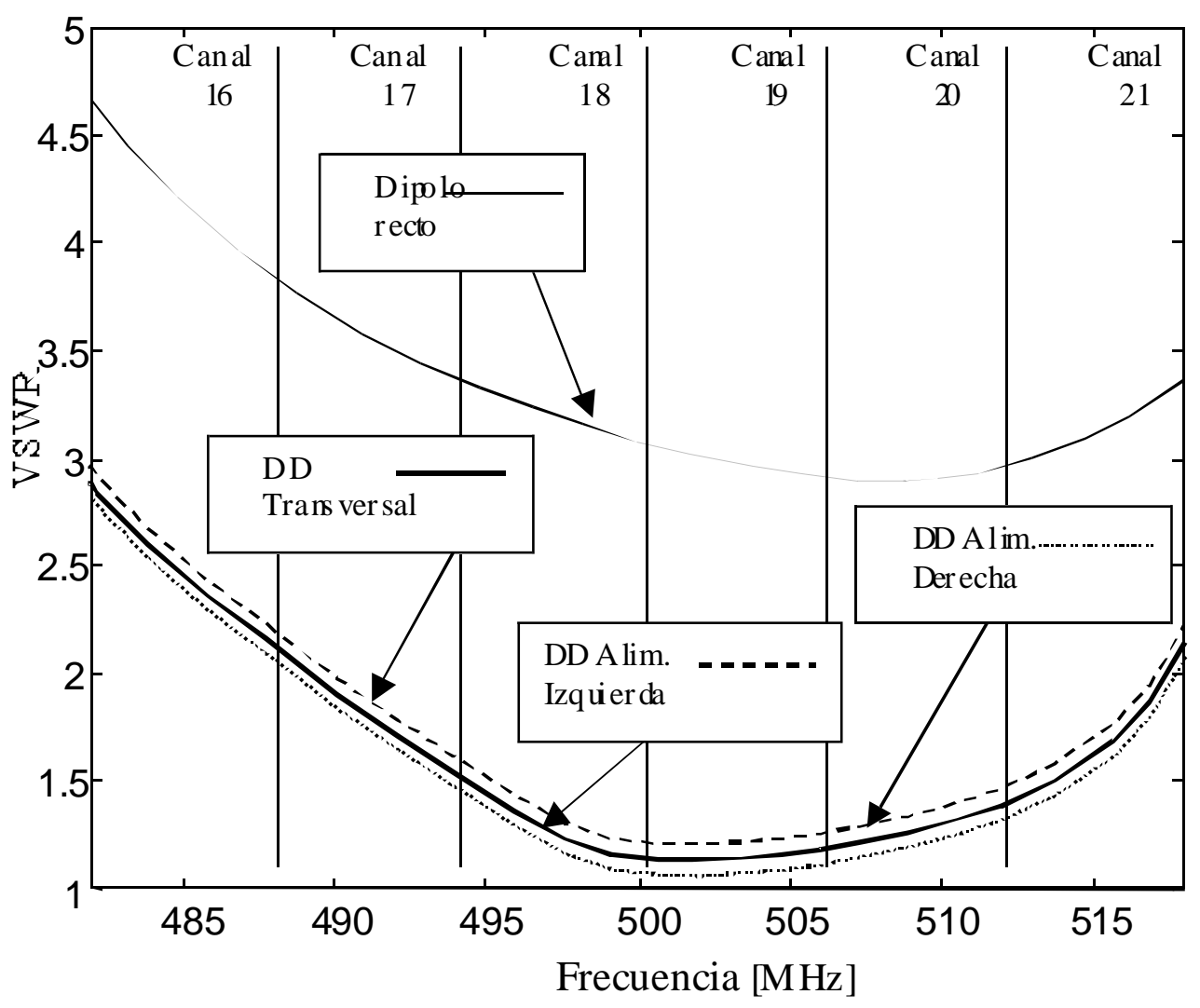

Figura 7. VSWR de arreglos Yagi de 15 elementos, considerando una alimentación con una línea de transmisión de $75 \Omega$. Véase la resistencia de entrada en estado de resonancia a $500 \mathrm{M} \mathrm{Hz}$ en la tabla 1

\section{c) Patrones de radiación}

La figura 8 muestra los patrones normalizados de radiación del campo eléctrico en el plano $\mathrm{E}$ y en el plano $H$ para arreglos de 5,7 y 15 elementos, utilizando un dipolo doblado transversal como alimentador (Figura $2 d$ ).

Cabe aclarar que el término "patrón de radiación" de uso común en México, es sustituido por "diagrama de radiación" en algunos países de habla hispana. Por razones de espacio, no se muestran los patrones o diagramas para el resto de los arreglos con dipolo doblado transversal descritos en la tabla 1, pero la siguiente discusión aplica también a ellos, ya que los datos fueron calculados y analizados por los autores. Al aumentar el número de elementos de los arreglos, pueden señalarse varias características interesantes: a) El ángulo entre la dirección de máxima radiación y la dirección de máxima intensidad del primer lóbulo lateral, disminuye al incrementar el número de elementos del arreglo, en ambos planos.

b) Como se esperaba, la directividad aumenta y el ancho de haz de media potencia (HPBW por sus siglas en inglés) disminuye, respectivamente en ambos planos, como se muestra en las figuras 8 y 9 .

c) Cuando el número de elementos es pequeño (5 ó 6), existe también una leve asimetría de los patrones con respecto al eje de simetría geométrica, 0 el eje $x$ en este caso particular, debido a que el punto de alimentación se encuentra desplazado una cierta distancia del origen del sistema de coordenadas (Figura $2 \mathrm{~d}$ ). 
Plano E

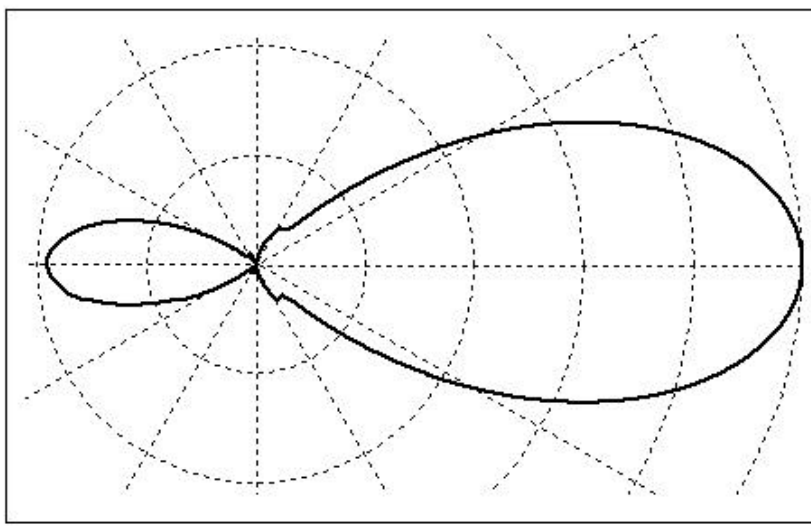

\section{Plano H}

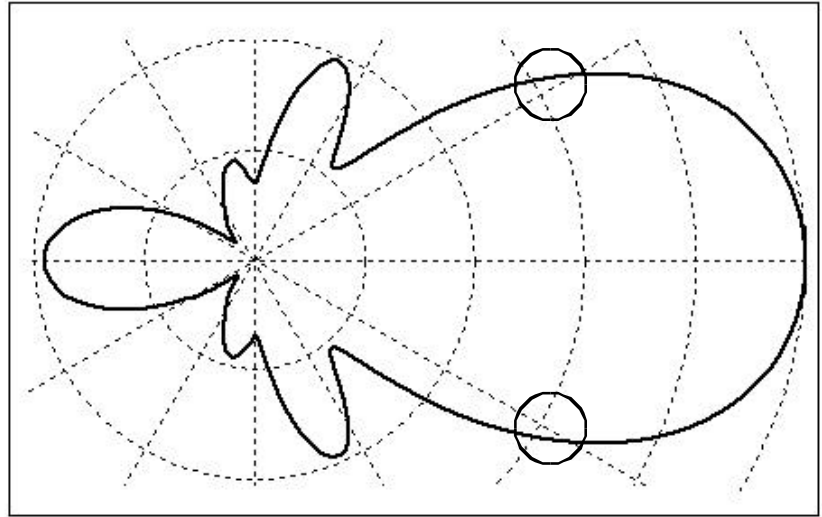

\section{5 elementos}
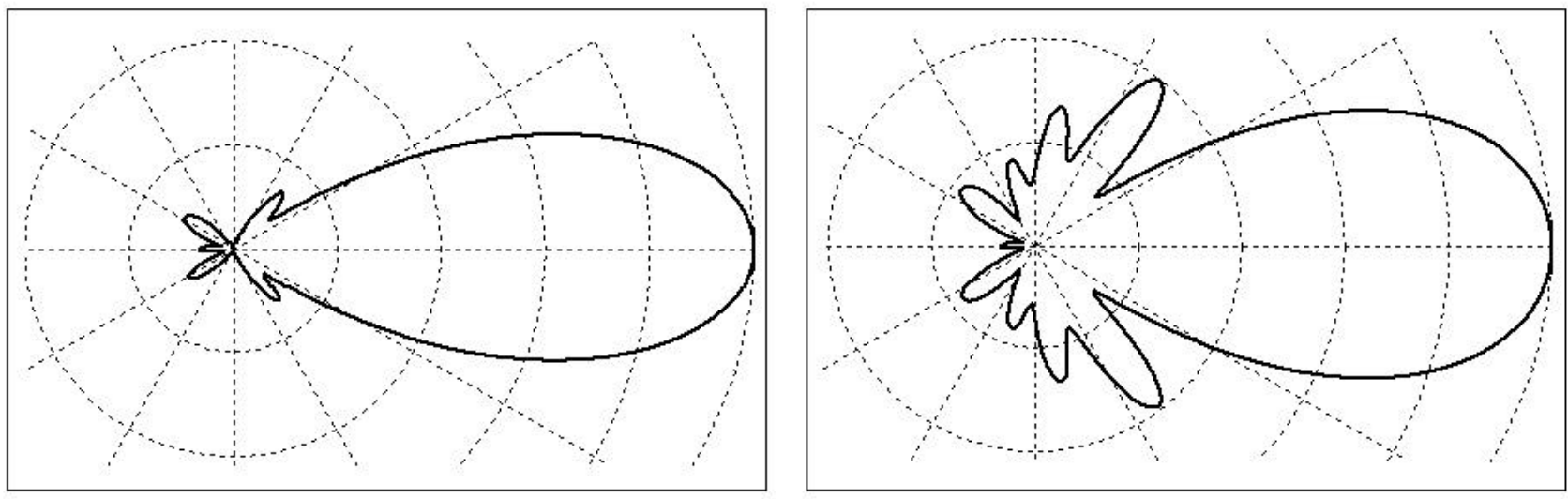

7 elementos
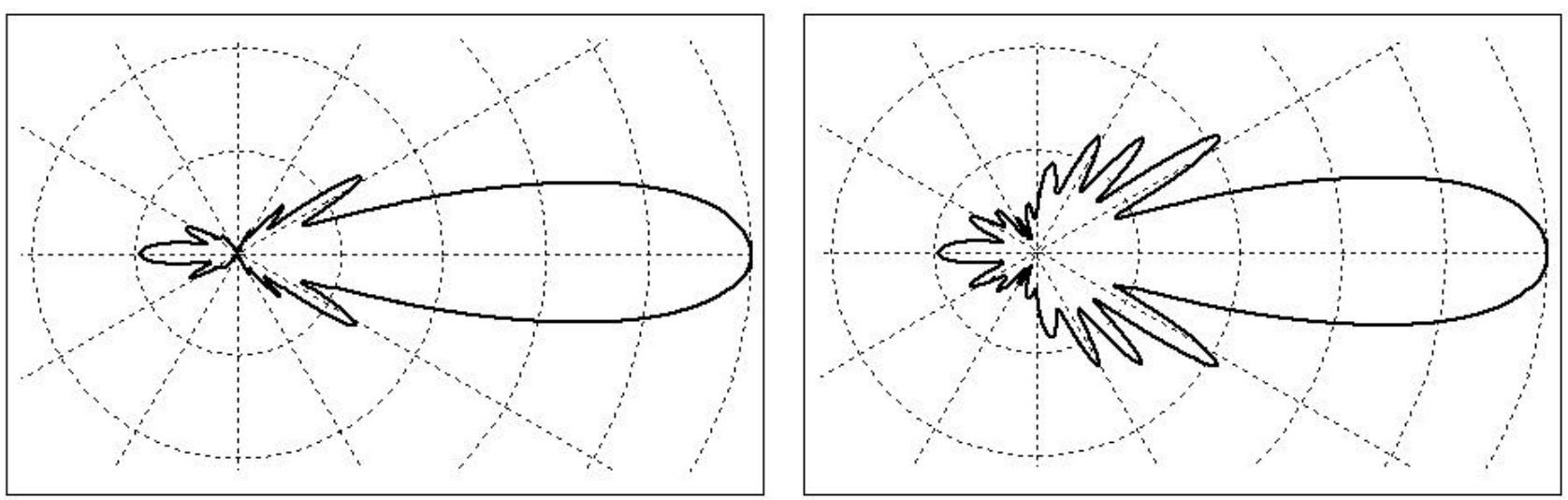

15 elementos

Figura 8. Patrones de radiación normalizados de campo eléctrico para arreglos Yagi, resonantes con 5,7 y 15 elementos. En todos los casos, el el emento alimentado es un dipolo doblado transversal con una separación $s_{d}$ entre sus dos brazos de $0.0833 \lambda$; el resto de los parámetros físicos están contenidos en la tabla $1 . N$ ótese la ligera asimetría en el plano $\mathrm{H}$, señalado con círculos en el arreglo de 5 elementos (Para datos numéricos más precisos, véase la figura 10) 
R. Neri-Vela, L.A. Valiente-Montaño y V. Hernández-Solís
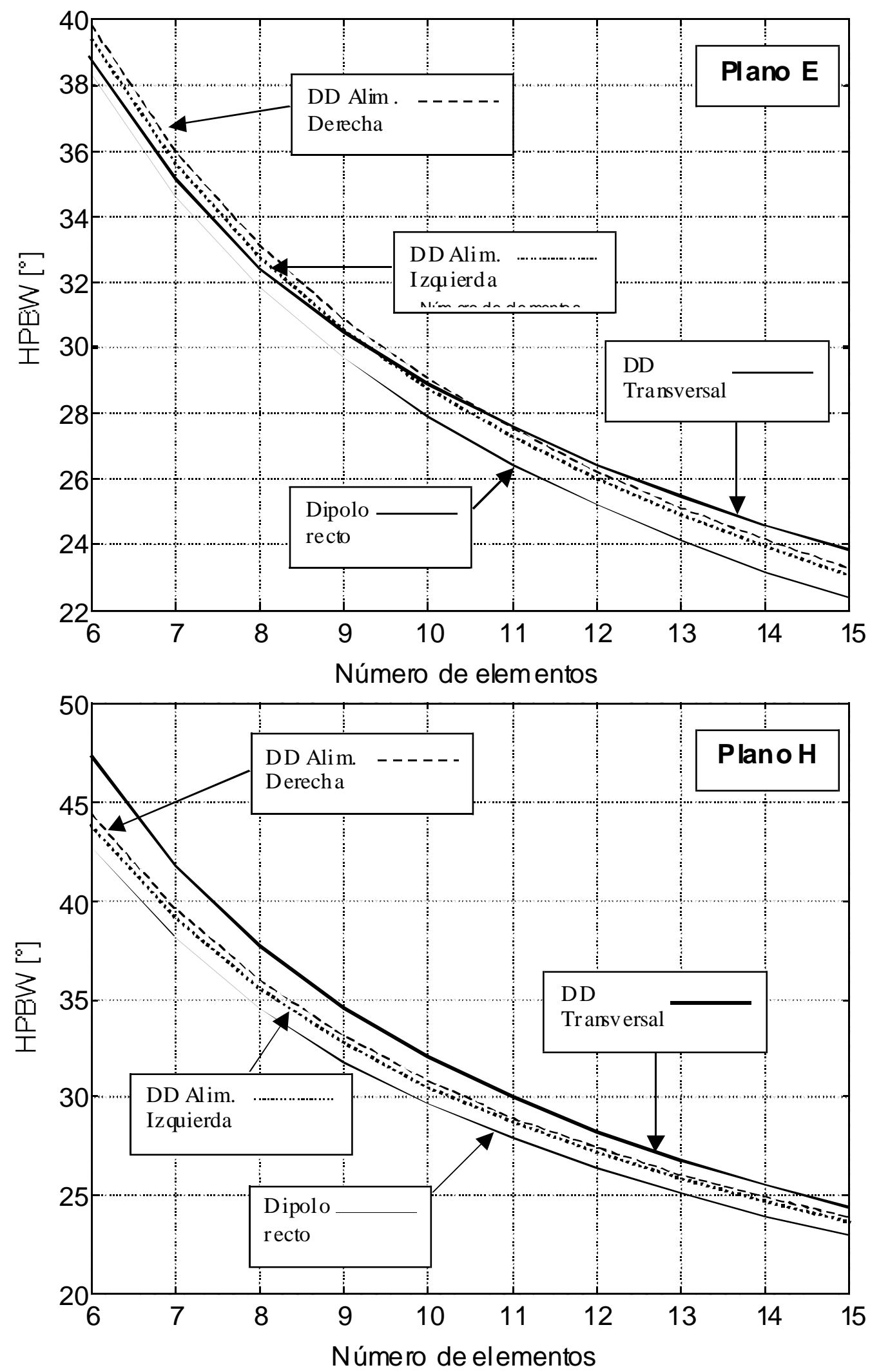

Figura 9. A ncho del haz de media potencia (H PBW por sus siglas en inglés) de los arreglos Yagi resonantes con las dimensiones presentadas en la tabla $1 s_{d}=0.0833 \lambda$ 
Comparación de la ganancia y otros parámetros de operación de antenas Yagi ...

Esta asimetría es del orden de medio grado y es más per cep ti ble en el plano $\mathrm{H}$, tal y como se señala con los dos pequeños círculos en la figura 8, pero prácticamente desaparece para arreglos de 70 más elementos. Para cuantificar mejor esta asimetría, la desviación en el plano x-z de la dirección de máxima radiación con respecto al eje $x$, denominado como ángulo de inclinación $\alpha$, se muestra graficado en la figura 10 para todos los arreglos considerados en este artículo con un dipolo doblado transversal como alimentador.

Pudiera pensarse que disminuir la distancia entre los brazos del dipolo doblado ayudaría a reducir dicha asimetría, y para analizar esta posibilidad se hicieron cálculos en arreglos resonantes utilizando una separación $s_{d}$ menor, de $0.03 \lambda$, ajustando la longitud del dipolo doblado para obtener resonancia y manteniendo fijos los parámetros restantes en la tabla 1. La gráfica correspondiente del ángulo de inclinación resultante se muestra también en la figura 10 , en la cual, contrariamente a la primera suposición, puede apreciarse que el ángulo de inclinación es ligeramente mayor (aunque por menos de una décima de grado) cuando se usa un dipolo doblado con una separacións $s_{d}$ menor, al menos para arreglos de 5 o más elementos; sin em bargo, para tres y cuatro elementos, la curva coincide con el criterio previamente asumido. En cualquier caso, el orden de magnitud de este ángulo es el mismo y las diferencias son muy pequeñas para cualquier número de elementos, así que lo importante es el comportamiento decreciente de la curva del ángulo de inclinación hasta llegar a niveles despreciables, en ambos casos, al incrementar el número de elementos. La desviación del haz prin cipal es tan insignificante que la potencia en la dirección del eje $x$ se reduce solamente en $10^{-3}$ a $10^{4} \mathrm{~dB}$ y, por lo tanto, cualquier desalineación en la instalación tendría efectos mayores; sin embargo, desde el punto de vista teórico es importante conocer este fenómeno, sin importar su magnitud.

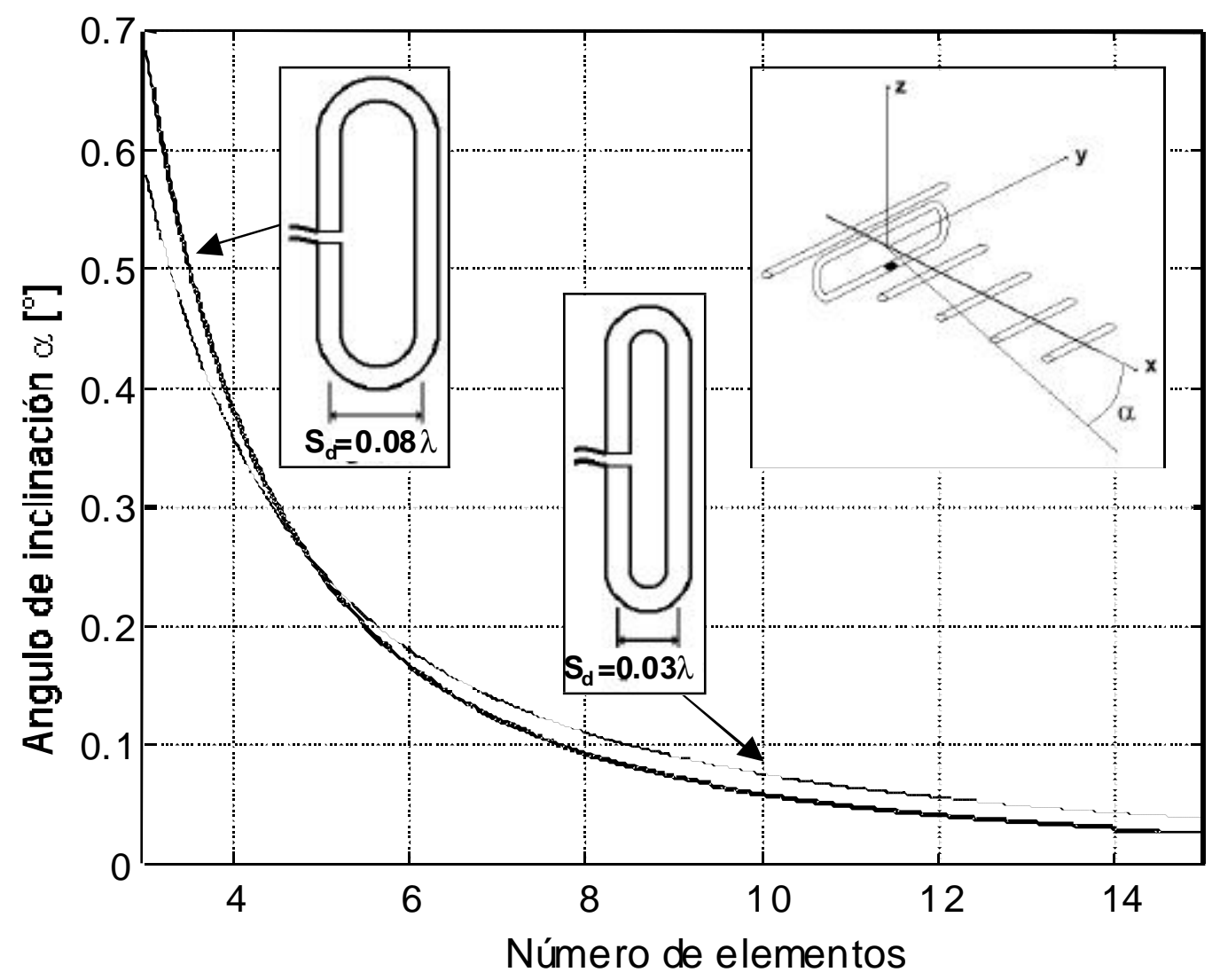

Figura 10. A rreglo Yagi alimentado con un dipolo doblado transversal. El ángulo de inclinación $\alpha$ está medido sobre el plano xz y muestra cómo la dirección de máxima radiación se desvía del eje $x$ 
R. Neri-Vela, L.A. Valiente-Montaño y V. Hernández-Solís

Puesto que los arreglos de las figuras $2 a, b$ y c son simétricos con respecto al eje $x$, no existe ángulo de inclinación $\alpha$ en ninguno de estos casos; sin embargo, las características (a) y (b) mencionadas al inicio de esta sección, concernientes a los patrones de radiación, sí aplican de igual forma.

Para hacer una comparación de las características de radiación de las cuatro configuraciones simuladas conviene referirse ahora a la figura 11 . Los autores seleccionaron el arreglo de 15 elementos como ejemplo, pero los detalles que a continuación se discuten son aplicables también en arreglos con un número menor de elementos, de acuerdo con los resultados y conclusiones obtenidos de los cálculos y gráficas pertinentes. La forma del patrón de radiación en el plano $\mathrm{E}$ es casi el mismo para las cuatro configuraciones, pero el arreglo alimentado con un dipolo recto tiene un primer lóbulo lateral ligeramente mayor que en aquellos arreglos alimentados con un dipolo doblado, ya sea coplanar o transversal; por otra parte, la antena alimentada con un dipolo recto proporciona un FBR ligeramente mayor. No obstante, en primera instancia y partiendo de la discusión an te rior, puede concluirse que, efectivamente, de acuerdo con lo expresado anteriormente por muchos autores (Balanis, 1997 y Gibilisco, 1999), las características de radiación no se ven afectadas realmente cuando se utiliza un dipolo doblado como alimentador o elemento excitado en lugar de uno recto; sin embargo, esto no es estrictamente correcto y sólo es válido en lo que se refiere a la forma gen eral del patrón de radiación. El ancho del haz de potencia media no es exactamente el mismo en los cuatro casos, tal y como se mostró en la figura 9, lo cual a su vez, revela que existen algunas pequeñas diferencias, quizá importantes, en la ganancia de la antena, lo cual se discutirá en la siguiente sección. Al observar los patrones en el plano $\mathrm{H}$ se obtienen conclusiones similares, con la excepción de que ahora el primer lóbulo lateral de mayor amplitud se encuentra en el arreglo alimentado con un dipolo transversal, aunque tiene una magnitud muy cercana a la del caso con un dipolo recto.

\section{d) G anancia}

Las ganancias de las cuatro configuraciones de arreglos Yagi analizadas, se muestran graficadas en la figura 12, en función del número de directores, con el propósito de comparar estas gráficas con las presentadas en la referencia (Green, 2000) para antenas típicas alimentadas con un dipolo recto. Puesto que esta referencia reporta la ganancia en $\mathrm{dB}$ relativos a la ganancia de un dipolo de longitud $\lambda / 2(\mathrm{dBd})$, a los valores calculados se les añadió $2.15 \mathrm{~dB}$ con el fin de graficar una aproximación en dBi fácil de comparar. Por supuesto, la gráfica de referencia (Green, 2000) no es una curva uni ver sal, ya que la ganancia verdadera depende de la combinación de longitudes y espaciamientos elegida, pero proporciona una idea aproximada de la ganancia esperada al incrementar el número de directores. Nótese que el comportamiento creciente y la pendiente de las curvas de ganancia con tra el número de directores concuerdan notablemente en todos los casos, y que es posible obtener un margen extra de ganancia de aproximadamente $1 \mathrm{~dB}$ si se utiliza un dipolo doblado en lugar de uno recto para cualquier número de directores. Esto demuestra que para los arreglos prácticos alimentados con un dipolo doblado, ya sea coplanar 0 transversal a los elementos parásitos, debe añadirse alrededor de $1 \mathrm{~dB}$ a los valores presentados en la literatura técnica que reporta resultados para arreglos alimentados con un dipolo recto, incluso si éste se encuentra en resonancia. Quizá una de las pocas excepciones que proporcionan algunos datos sobre antenas Yagi alimentadas con un dipolo doblado transversal es la referencia (Viezbicke, 1968) y los autores han aprovechado algunos de estos datos para verificar los valores calculados con el método de momentos. Asimismo, algunos ejemplos de arreglos Yagi optimizados han sido tomados de esta fuente y publicados en (Stutzman et al., 1998) y (Johnson, 1993) para arreglos con 3, 4 y 10 directores, cuyas ganancias correspondientes son reportadas como 9.2, 10.2 y $12.25 \mathrm{~dB}$, respectivamente, relativos a un dipolo recto de media longitud de onda; en todos los casos, el elemento alimentado es un dipolo doblado, cuya longitud fue ajustada empíricamente para lograr un VSWR mínimo en la frecuencia de diseño, la longitud de todos los directores es aproximadamente de $0.4 \lambda$ y la separación entre ellos es de alrededor de $0.25 \lambda$. Al añadir a estos valores $2.15 \mathrm{~dB}$, que es la ganancia del dipolo recto de media longitud de onda, se obtiene la ganancia correspondiente en 
Comparación de la ganancia y otros parámetros de operación de antenas Yagi ...

Plano E
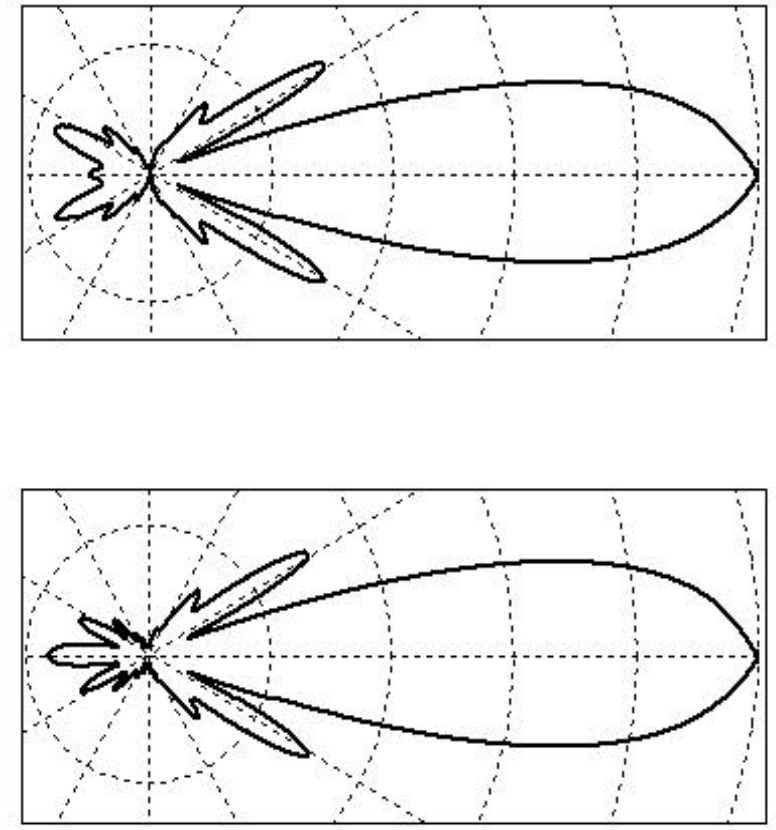

(b)

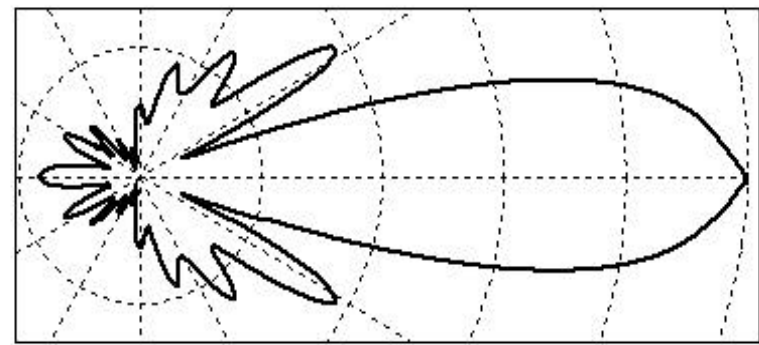

(c)

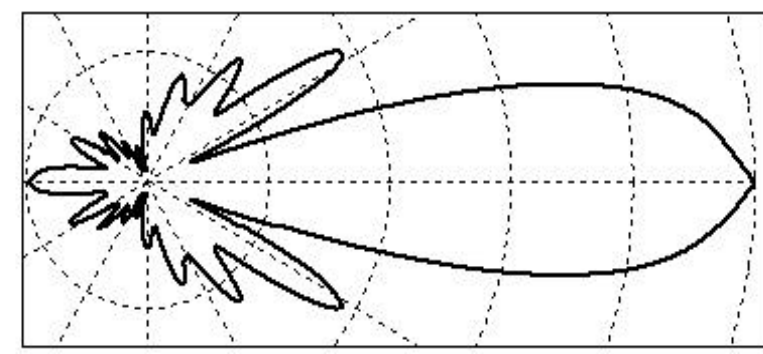

(d)

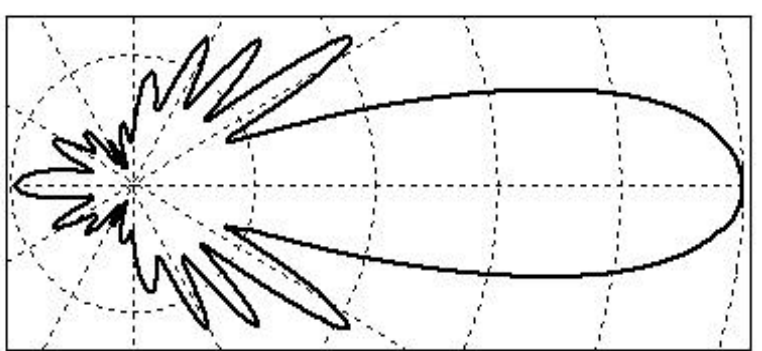

Figura 11. Patrones de radiación normalizados de campo eléctrico para arreglos Yagi resonantes con 15 elementos, alimentados con: a) Un dipolo recto, b) Un dipolo doblado coplanar alimentado en su brazo izquierdo, c) Un dipolo doblado coplanar alimentado en su brazo derecho; y d) Un dipolo doblado transversal 
R. Neri-Vela, L.A. Valiente-Montaño y V. Hernández-Solís

dBi marcada en la figura 12 por medio de círculos pequeños, lo cual comprueba la validez de los resultados presentados en este artículo. De hecho, se comprueba también que el utilizar una separación ligeramente mayor entre los directores, de $0.4 \lambda$ como se establece en la tabla 1 , no altera la operación óptima en términos de ganancia, de acuerdo con lo reportado por (Viezbicke, 1968).

Finalmente, las figuras 13 y 14 muestran las variaciones de la ganancia en función de la frecuencia, manteniendo los mismos parámetros físicos en los arreglos de 5 y 15 elementos, respectivamente, de forma sim i lar al cálculo de las graficas de VSWR mostradas en las figuras 6 y 7 . Se observa que en una banda de varios ca na les de TV en UHF, los arreglos con dipolo doblado siguen manteniendo una ganancia aproximadamente $1 \mathrm{~dB}$ mayor que la de la antena alimentada con un dipolo recto.

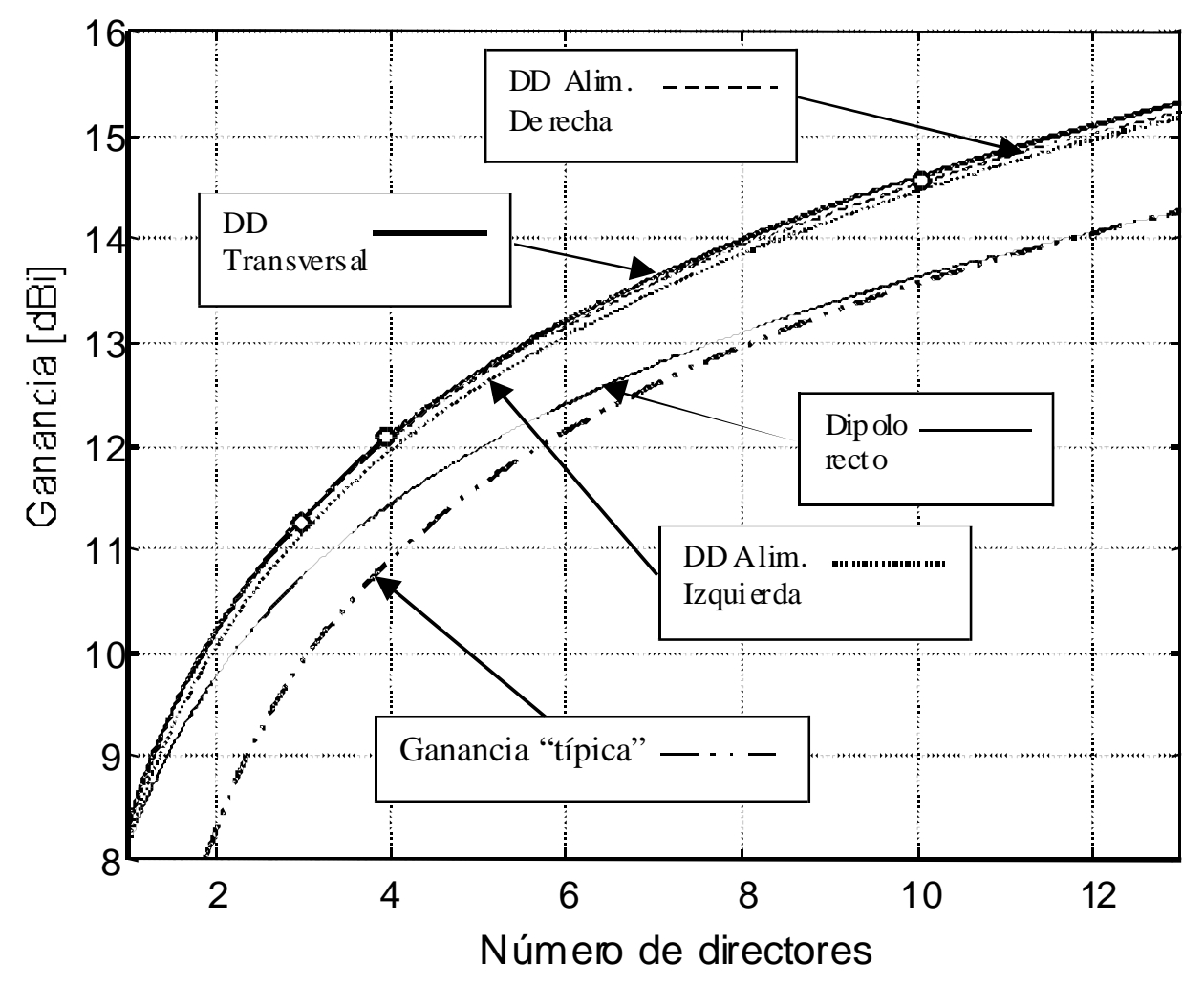

Figura 12. Ganancia de los arreglos Yagi resonantes presentados en este artículo, comparada con los valores considerados "típicos" para un amplio márgen de longi tudes y espaciamientos, usando un dipolo recto como alimen tador ( $\mathrm{G}$ reen, 2000$).$

$\mathrm{N}$ óten se los pequeños círculos ${ }^{\circ}$ para 3, 4 y 10 directores, que son datos experimentales (Viezbicke, 1968) usando un dipolo doblado como alimentador y que comprueban la validez del método de momentos 
Comparación de la ganancia y otros parámetros de operación de antenas Yagi ...

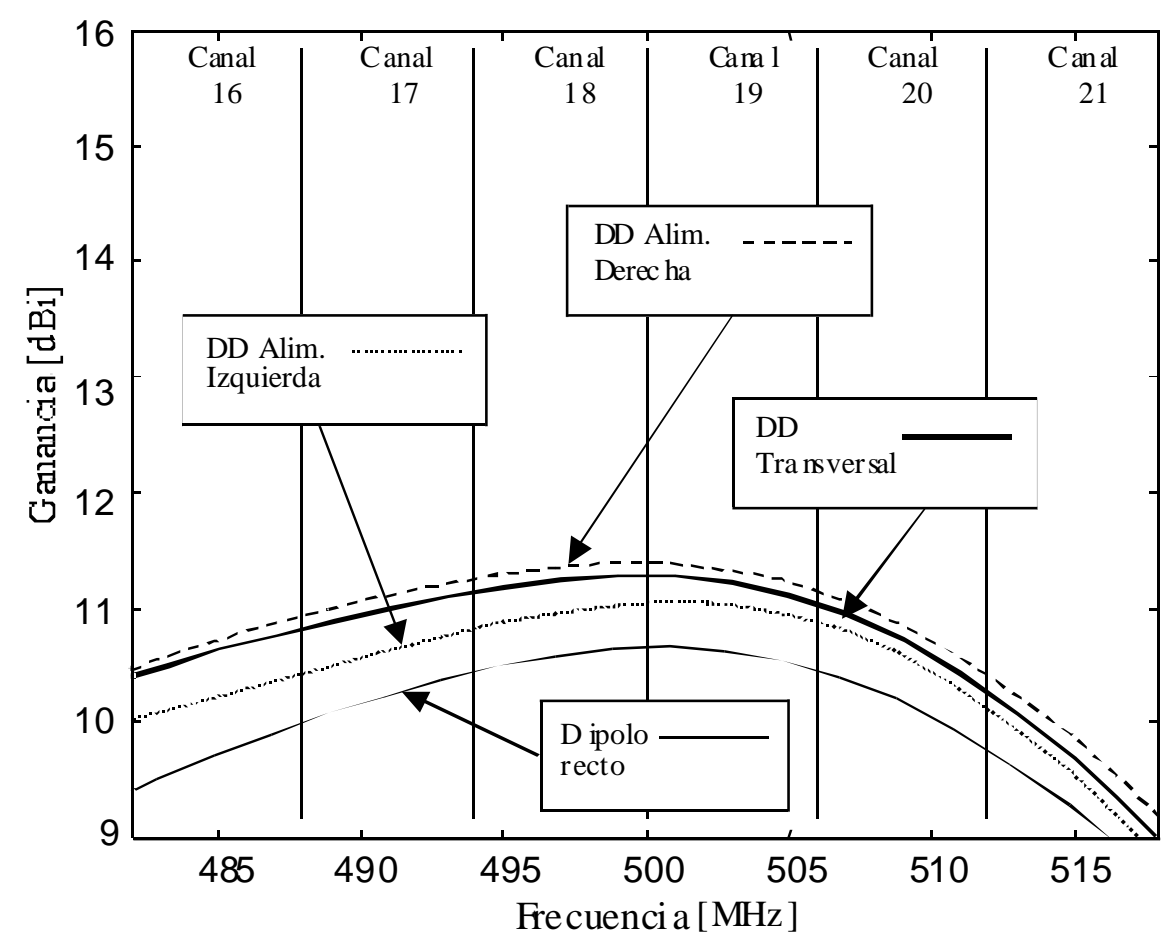

Figura 13. Ganancia de arreglos Yagi de 5 elementos, conservando fijas las dimensiones físicas de cada antena e iguales a las del estado de resonancia en $500 \mathrm{MHz}$

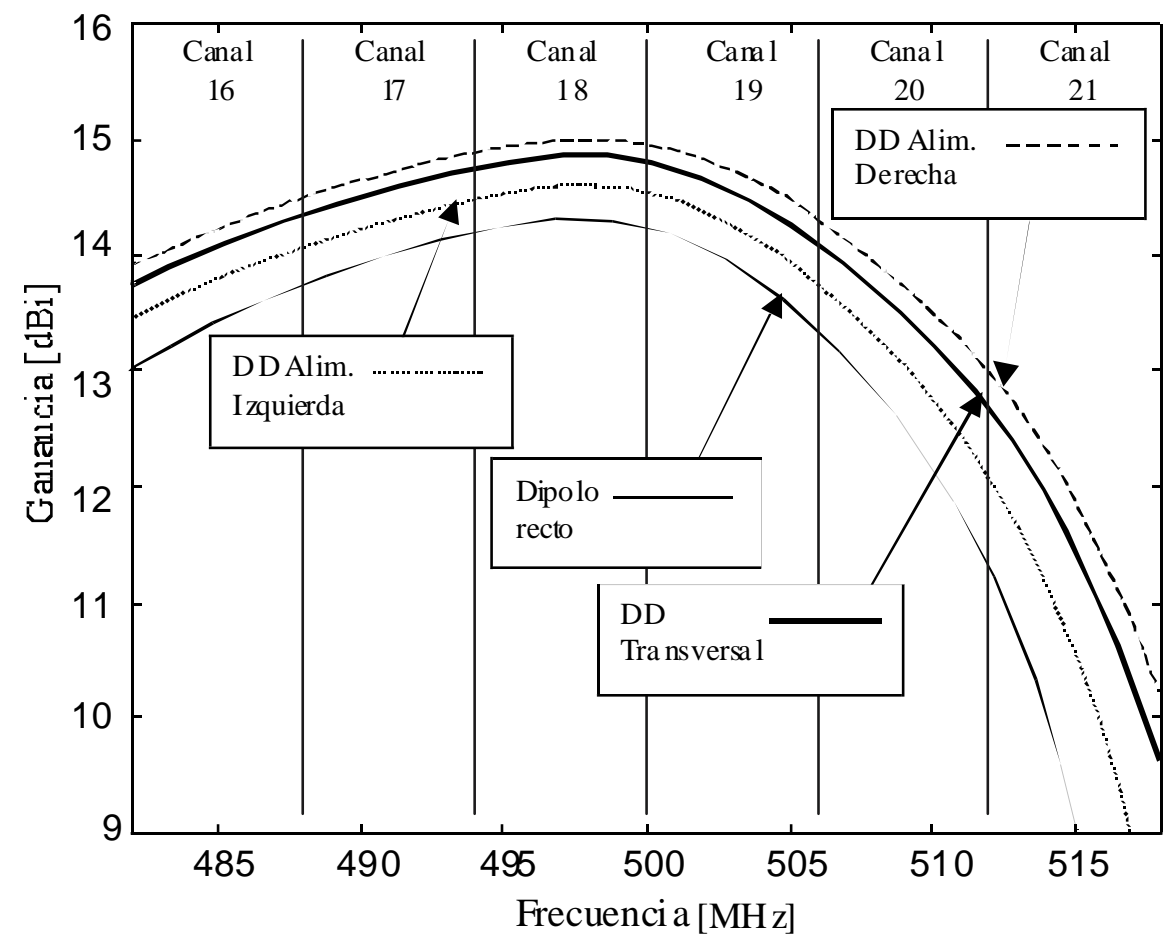

Figura 14. Ganancia de arreglos Yagi de 15 elementos, conservando fijas las dimensiones físicas de cada antena e iguales a las del estado de resonancia en $500 \mathrm{M} \mathrm{Hz}$ 
R. Neri-Vela, L.A. Valiente-Montaño y V. Hernández-Solís

\section{Conclusiones}

Los resultados presentados en este trabajo amplían considerablemente la información disponible sobre el funcionamiento de distintos tipos de antenas Yagi, según su forma de alimentación 0 excitación. Se concluye que las características de operación de antenas Yagi alimentadas con un dipolo doblado no son exactamente iguales a las de antenas Yagi alimentadas con un dipolo recto. Aunque la forma de los patrones de radiación es muy similar para ambos casos, la ganancia y el ancho del haz principal son diferentes. Una Yagi alimentada con un dipolo doblado tiene $1 \mathrm{~dB}$ más de ganancia que una antena similar alimentada con un dipolo recto. Las principales diferencias de operación entre ambas antenas se muestran concentradas en la tabla 2.

Tabla 2. Conclusiones sobre las diferencias entre la operación de arreglos Yagi resonantes, alimentados con dipolos sencillos o doblados

\begin{tabular}{|c|c|c|c|c|}
\hline $\begin{array}{l}\text { Tipo de } \\
\text { alimentación }\end{array}$ & $\underset{[\mathrm{dBi}]}{G \text { Ganancia }}$ & Corrientes & $\begin{array}{l}\text { Ancho de } \\
\text { haz de } \\
\text { media } \\
\text { potencia } \\
\text { (HPBW) } \\
\text { Plano H }\end{array}$ & $\begin{array}{c}\text { Ancho de } \\
\text { haz de } \\
\text { media } \\
\text { potencia } \\
\text { (HPBW } \\
\text { Plano }-\end{array}$ \\
\hline $\begin{array}{l}\text { Dipolo } \\
\text { sencillo }\end{array}$ & G & $\begin{array}{l}\text { Máxima en } \\
\text { el dipolo } \\
\text { alimentado }\end{array}$ & HPBW & HPBW \\
\hline $\begin{array}{l}\text { Dipolo } \\
\text { doblado } \\
\text { coplanar } \\
\text { (alim. } \\
\text { izquierda) }\end{array}$ & $G+0.6$ & $\begin{array}{l}\text { Máxima en } \\
\text { el reflector }\end{array}$ & $\begin{array}{l}\text { HPBW } \\
+1.1^{\circ}\end{array}$ & $\begin{array}{l}\text { HPBW } \\
+0.8^{\circ}\end{array}$ \\
\hline $\begin{array}{l}\text { Dipolo } \\
\text { doblado } \\
\text { coplanar } \\
\text { (alim. } \\
\text { derecha) }\end{array}$ & $G+1$ & $\begin{array}{l}\text { Máxima en } \\
\text { el reflector }\end{array}$ & $\begin{array}{l}\text { HPBW } \\
+1.2^{\circ}\end{array}$ & $\begin{array}{l}\text { HPBW } \\
+1.0^{\circ}\end{array}$ \\
\hline $\begin{array}{c}\text { Dipolo } \\
\text { doblado } \\
\text { transversal }\end{array}$ & $G+1$ & $\begin{array}{l}\text { Máxima en } \\
\text { el reflector }\end{array}$ & $\begin{array}{l}\text { HPBW } \\
+2.8^{\circ}\end{array}$ & $\begin{array}{l}\text { HPBW } \\
+0.4^{\circ}\end{array}$ \\
\hline
\end{tabular}

\section{Referencias}

Balanis C.A. (1997). Antenna Theory, Analysis and Design. 2 a edición, Wiley.

Cardama A., Jofre LI., Rius J.M., Romeu J., Blanch S. y Ferrando M. (2004). Antenas. 2a edición, Alfaomega.

Chen C.A. and Cheng D.K. (1975). Optimum Element Lengths for Yagi-Uda Arrays. IEEE Trans. Antennas and Propagation, Vol. AP-23, No. 1, pp. 8-15.
Cheng D.K. and Chen C.A. (1973). Optimum Element Spac ings for Yagi-Uda Arrays. IEEE Trans. Antennas and Propagation, Vol. AP-21, No. 5, pp. 615-623.

Combes P.F. (1997). Circuits Passifs, Propagation, Antennes, Micro-ondes. Vol. 2. Dunod, Francia.

Ehrenspeck H.W. and Poehler H. (1959). A N ew Method for Obtaining Maximum Gain from Yagi Antennas. IRE Trans. Antennas and Proppgation, Vol. AP-7, p. 379.

Gibilisco S. (1999). Handbook of Radio and Wireless Tech nology . McGraw-Hill.

Green D.C. (2000). Radio Communication, 2a edición, Longman, pp. 229-233.

Griffiths J. (1987). Radio Wave Propagation and Antennas, AnIntroduction. Prentice-Hall.

Harrington R.F. (1968). Field Computation by $M$ oment M ethods, Macmillan.

Hernández V., Valiente L.A. and Neri R. (2001). Input Impedance, Current Distributions, and Radiation Properties of Thick and Widely Spaced Folded Dipoles. Electromagnetics, Vol. 21, pp. 435-449.

Jasik H. (1961). Antenna Engineering $H$ andbook. McGraw-Hill.

Johnson R.C. (1993). Antenna Engi neering $\mathrm{H}$ andbook, 3a edición. McGraw-Hill, pp. 29-16 a 29-21.

King R.W.P. (1989). Supergain Antennas and the Yagi and Circular Arrays. IEEE Trans. Antennasand Prop a ga tion, Vol. 37, No. 2, pp. 178-186.

Kraus J.D. (1988). Antennas. 2a edición. McGraw-Hill.

Landeros S., Bisiacchi G., Pulinets S., Neri R. y De la Rosa S. (2005). Predicción de terremotos con un nanosatélite mexicano. Ciencia y Desarrollo. Conacyt, Vol. 31, No. 188, pp. 26-29.

Mailloux R.J. (1966). The Long Yagi-Uda Array. IEEE Trans. Antennas and Propagation, Vol. AP-14, pp. 128-137.

Neri R. (1980). Self and Mutual Impedances Between Sub-elements of Dipole Antennas: Contributions from Currents and Charges. Int. J. Elec tronics, Vol. 48, pp. 435-442.

Neri R., Hernández V. and Valiente L.A. (2003). Compar ison of V-Grid Reflec tors Fed with a 
Comparación de la ganancia y otros parámetros de operación de antenas Yagi ...

Single Dipole or with Yagi Antennas. Electromagnetics, Vol. 23, No. 6, pp. 481-490.

Neri R., Valiente L.A. y Hernández V. (2005). Comparación analítica de arreglos de antenas Yagi por los métodos de momentos y multiplicación de patrones. Ingeniería Investigación y Tecnología, UNAM, Vol. VI, No. 2, Abril-Junio.

Stutzman W.L. and Thiele G.A. (1998). Antenna Theory and Design. 2 a edición, Wiley.

Thiele G.A. (1969). Analysis of Yagi-Uda Type Antennas. Trans. Antennas and Propagation, Vol. AP-17, pp. 24-31.
Tsai L.L. and Smith Ch.E. (1978). Moment Methods in Electromagnetics for Under graduates. IEEE Trans. on Education, Vol. E-21, No. 1, pp. 14-22.

Viezbicke P.P. (1968). Yagi A ntenna D esign, NBS Tech. Note 688, National Bureau of Standards, Wash ington.

Wang J.J.H. (1990). Generalised Moment Methods in Electromagnetics. IEE Proceedings, Vol. 137, Pt. H, N 0. 2, pp. 127-132.

\section{Semblanza de los autores}

Rodolfo N eri-Vela. En 1975, obtuvo su título de ingeniero mecánico-electricista en el área de comunicaciones y electrónica por la Facultad de Ingeniería de la UNAM. En 1976, recibió el grado de maestría en sistemas de telecomunicaciones otorgado por la Universidad de Essex, Reino Unido. En 1979, alcanzó el grado de doctorado en electromagnetismo aplicado por parte de la Universidad de Birmingham, también en el Reino Unido. En 1985, se convirtió en el prime rastronauta de México, al participar en la misión 61-B de la NASA de los EU y orbitar la Tierra 109 veces. Es profesor e nuvestigador del Departamento de Telecomunicaciones de la Facultad de Ingeniería de la UNAM desde hace 24 años y pertenece al Sistema Nacional de Investigadores del CONACYT.

Luis A lán Valiente-M ontaño. Obtuvo su título en el año 2000 como ingeniero en telecomunicaciones por la Facultad de Ingeniería de la UNAM . En el 2003, recibió el grado de maestría en ingeniería de comunicaciones otorgado por el Instituto de Ciencia y Tecnología de la Universidad de Manchester (UMIST), Reino Unido. Actualmente está becado por el CONACYT y realiza sus estudios de doctorado en la misma Universidad de Manchester, en el área de microscopía por microondas en el campo cercano.

Vladimir Hernández-Solís. Se tituló como ingeniero en telecomunicaciones en el 2000, en la Facultad de Ingeniería de la UNAM. En el año 2003, recibió el grado de maestría en ingeniería de comunicaciones otorgado por el Instituto de Ciencia y Tecnología de la Universidad de Manchester (UMIST), Reino Unido. Tiene experiencia en arreglos adaptivos para redes celulares CDMA, antenas y óptica no lineal. Actualmente realiza sus estudios de docto rado en el Centro de Investigaciones en Optoelectrónica de la Universidad de Southampton, Reino Unido, en el área de amplificadores de fibra de alta potencia. 